\title{
Modelo para reducción de mora quirúrgica en cirugías selectivas del Hospital El Progreso, Yoro
}

\author{
Mario José Sánchez ${ }^{1}$, M.D., M.Sc. y Luis Fernando Enamorado, M.Sc. \\ UNITEC, San Pedro Sula, Honduras.
}

(Recibido: Agosto, 2013 y Aceptado para publicación: Noviembre, 2015)

\begin{abstract}
Resumen:
La presente investigación se desarrolló en el Hospital de El Progreso, Yoro, Honduras. Datos estadísticos han arrojado que en la actualidad la mora quirúrgica de cirugía selectiva es de 120 días, lo que contrasta con los 30 días usados como indicador meta por parte del Ministerio de Salud de Honduras y recomendado por la Organización Mundial de la Salud (OMS). En vista de esta problemática se propone un modelo a través de simulación, que evalúe formas de disminuir dicha mora quirúrgica. Para el diseño de la investigación se usó un enfoque mixto dominante cuantitativo. Para alimentar el software de simulación Flexsim y corroborar el modelo actual de forma simulada, se construyó una bitácora de cirugías del 2012, gráficos de caja y bigote, pruebas de ANOVA y de hipótesis para cada cirujano (A, B y C) en las seis intervenciones quirúrgicas más frecuentes según diagrama de Pareto. Al tener el modelo actual validado, se recrearon cinco tipos de escenarios. Se eligió el primero, ya que es el que implica menor costo con alto impacto, obteniendo una disminución de la mora quirúrgica a solo 38 días. Por lo que se recomienda implementar el escenario número uno para reducir la mora y cumplir con el presupuesto del hospital.
\end{abstract}

Palabras Claves: Bitácora de cirugía, Escenarios, Indicador, Mora Quirúrgica.

\begin{abstract}
:
This research was conducted at El Progreso Hospital in Yoro, Honduras. Statistical data shows that the current surgical delay time is 120 days, compared to the 30 day recommendation made by the World Health Organization and the Honduran government. In light of this problem, a simulation model has been constructed to evaluate ways of decreasing such waiting periods. For the simulation design the study used a quantitative-dominant mixed model. Data was given to the Flexism simulation software to corroborate the parameters used in the simulation, the study constructed a log of surgeries from the year 2012, conducted boa-and-whiskers plots, ANOVA tests and hypothesis tests for each of the surgeons (A,B, and C) in the six most frequent surgical interventions according to the Pareto Diagram. Once the actual model had been validated, 5 types of scenarios were re-created. The first scenario was chosen because it was found to have the most impact and the least cost, resulting in reduction of the waiting time to only 38 days. Therefore, it is recommended to implement the first scenario to reduce the waiting time and to stay within hospital budget.
\end{abstract}

Keywords: Surgery log, Settings, Display, Surgical delay.

\section{Introducción}

En la actualidad, los hospitales nacionales continúan sin contar con soluciones eficientes, que permitan utilizar de la mejor forma los recursos asignados por el Ministerio de Salud y que proporcionen un buen servicio al usuario final. Un problema particular es lograr que cada paciente pueda tener su intervención de cirugía selectiva dentro de los siguientes

\footnotetext{
${ }^{1}$ Autor para correspondencia. Email: sanchez_andara@hotmail.com

* Los autores agradecen a los ingenieros Jared R. Ocampo y Abel Salazar, quienes les asesoraron y dieron importantes aportes en la metodología usada y el modelo de simulación implementado para desarrollar este trabajo de investigación y su correspondiente artículo.
} 
treinta días de ser evaluado y diagnosticado por el médico especialista (cirujano general), para evitar un agravamiento de su patología. El sistema sanitario de Honduras, es regulado por la Secretaría de Salud, responsable de atender a la población nacional. Por ley, no debe cobrar por sus servicios a la población y darlos de forma gratuita, aunque en la práctica, los pacientes son quienes tienen que comprar los medicamentos, pagar por análisis de laboratorio y por insumos de cirugía que no están disponibles en el hospital.

El Hospital El Progreso, desde su fundación en 1983, se ha mantenido relativamente constante en la cobertura de las cuatro áreas básicas (medicina interna, ginecología, pediatría y cirugía), siendo cirugía general, la que actualmente tiene menos cobertura. El hospital cuenta con tres cirujanos, dos en la jornada matutina y uno en la vespertina (con modalidad de contrato) y cuenta con dos cirujanos de guardia nocturna (modalidad permanente).

La investigación se centra en los tres cirujanos del día, ya que solo ellos tienen consulta externa para poder programar las cirugías selectivas en este centro asistencial. El departamento de estadística del Hospital El Progreso, no cuenta, hasta este momento, con datos de cirugías selectivas del 2011 hacia atrás, ya que el equipo de cómputo sufrió daños sin poder corregirlo aún, pero si cuenta con información del total de consultas realizadas por el servicio de cirugía general.

Dada la situación, se pretende generar un modelo matemático de simulación que permita experimentar de forma virtual con escenarios que busquen reducir la mora quirúrgica actual de las cirugías selectivas que es de 120 días en el Hospital El Progreso y acercarse así al indicador meta que tiene la Organización Mundial de la Salud (OMS) de 30 días. La presente investigación busca demostrar que mejorando el sistema actual, haciendo un uso más eficiente del recurso humano y material, implementando los cambios necesarios y aplicando una reingeniería en el proceso de cirugía en el hospital de El Progreso, se puede alcanzar los indicadores de la OMS. Esto significa, como se citó anteriormente, reducir el tiempo de mora de los 120 días actuales a los 30 días sugeridos y estandarizados por esta organización internacional.

Con todo esto se marcaría un precedente en el sistema de salud, se desarrollaría un proceso que puede ser implementado en todos los centros hospitalarios del país y sobre todo se mejoraría la calidad y la expectativa de vida de las personas que visitan y dependen del Hospital El Progreso.

\section{Revisión de literatura}

El sistema de salud de Honduras está compuesto por un sector público y otro privado. El sector público incluye a la Secretaría de Salud (SS) y al Instituto Hondureño de Seguridad Social (IHSS). Además de otras instituciones públicas que administran regímenes especiales de aseguramiento (Fuerzas Armadas) o atienden a poblaciones específicas. La SS ofrece atención a toda la población en sus propias instalaciones y con sus propios médicos y enfermeras, pero se estima que sólo $60 \%$ de los hondureños hacen uso regular de estos servicios.

El hospital de área de El Progreso, es parte del sistema de salud público de Honduras y brinda servicios en cuatro áreas básicas (medicina interna, ginecología, pediatría y cirugía general). Más recientemente se cuenta con neurocirugía, otorrinolaringología, psiquiatría 
y gastroenterología. Actualmente el hospital cuenta con 350 empleados de los cuales 250 son con plaza permanente y 100 por contrato.

Estos empleados rotan cada cuatro años por determinaciones políticas. Al lugar llegan enfermos de El Progreso, Santa Rita, El Negrito, Morazán, Yoro, con una población estimada de 250,000 habitantes y también algunas personas de los departamentos de Cortés y Atlántida.

Cada intervención quirúrgica debe ser realizada por cirujanos especialistas, los cuales son asignados por El Hospital El Progreso. Como todo hospital público del país, se atiende a toda la población que llega. El área de emergencia es el lugar de donde se genera el ingreso de pacientes con características clínicas diferentes a los pacientes programados para cirugía selectiva.

Los pacientes de emergencia son pacientes que tienen una patología que no se puede diferir a la consulta externa. Igualmente esta patología puede ser secundaria a traumas tales como, accidente automotor, heridas penetrantes, entre otras y secundaria a urgencias quirúrgicas; apendicitis aguda, perforación de víscera hueca y hernias estranguladas (Schwartz, 2010). Este tipo de intervenciones médicas no pueden ser interrumpidas una vez iniciadas, requieren de pabellones que cumplan con necesidades particulares de equipamiento, y se debe disponer de personal de apoyo: enfermeras, anestesistas, auxiliar de anestesia, recuperadoras y en algunos casos, uno o dos cirujanos asistentes (Rojas y Vergara, 2012).

Para poder llevar a cabo una cirugía dentro del quirófano del Hospital El Progreso se requiere por lo menos de un anestesista, dos instrumentistas, un cirujano general, un ayudante y una aseadora. El paciente al terminar la cirugía pasa a la sala de recuperación donde, por lo general, se encuentra de una a dos horas monitorizado. Si no se detecta ninguna contraindicación es trasladado a la sala de cirugía en el área de hospitalización.

\section{Metodología}

El diseño de la investigación fue mixto. En este proceso se recolectó, analizó y vinculó datos cuantitativos y cualitativos en un mismo estudio para responder a un planteamiento del problema (Hernández, Fernández, \& Baptista, 2006). Siguiendo la línea de la investigación cuantitativa se determina como no experimental, transversal y descriptiva. A su vez es cualitativa ya que se realizaron entrevistas a personal clave para la obtención de información verídica en la formulación de soluciones del problema.

Las hipótesis de la investigación son las siguientes:

Hi: El modelo de simulación no refleja la realidad de lo que está pasando en el sistema real en donde una mora de 120 días (es válida) y por consiguiente no puede utilizarse como predictor para los diferentes escenarios y planteamientos de solución.

Ho: El modelo de simulación refleja la realidad de lo que está pasando en el sistema real en donde una mora de 120 días (es válida) y por consiguiente puede utilizarse como predictor para los diferentes escenarios y planteamientos de solución.

Las variables de investigación son: mora quirúrgica (dependiente) y las variables independientes: cirujano general, insumos, asambleas informativas, tiempo programado 
de cirugía, personal técnico, personal de anestesia, cancelación en pabellón y cirugía de emergencia.

Para esta investigación se tomaron en cuenta todas las cirugías selectivas realizadas en el 2012, en el Hospital El Progreso. En ese año hubo 714 cirugías de las cuales 264 fueron selectivas. Tomando estas cirugías, se calculó el tamaño muestral probabilístico con un nivel de confianza del 95\%, un error estándar de 5\% y una proporción característica del $50 \%$, para obtener información confiable y desarrollar este proyecto. El cálculo de la muestra se realizó con la siguiente fórmula (Hernández, Fernández, \& Baptista, 2006):

$$
n=\frac{Z^{2} p q N}{N \mathrm{e}^{2}+\mathrm{Z}^{2} \mathrm{pq}} n=\frac{1.96^{2}(0.5)(0.5)(462)}{462\left(0.05^{2}\right)+\left(1.96^{2}\right)(0.5)(0.5)}=210 \text { cirugías selectivas }
$$

Donde: $\mathrm{N}=$ Tamaño de la población; $\mathrm{Z}=$ Nivel de confianza; $\mathrm{p}=$ Prevalencia esperada del parámetro que se ha de estimar; $q$ = Prevalencia esperada del parámetro que no se ha de estimar y e $=$ error estándar

El equipo principal que se utilizó para esta investigación fue la computadora, ya que se corrieron los diferentes escenarios del modelo generado utilizando el software de simulación de eventos discretos Flexsim. Se realizó un diagrama de Pareto ordenando todas las cirugías obtenidas por la bitácora de cirugías. Este ordenamiento se hizo comenzando con las cirugías con más incidencias hasta las menos frecuentes. A continuación se muestra un gráfico de las principales cirugías.

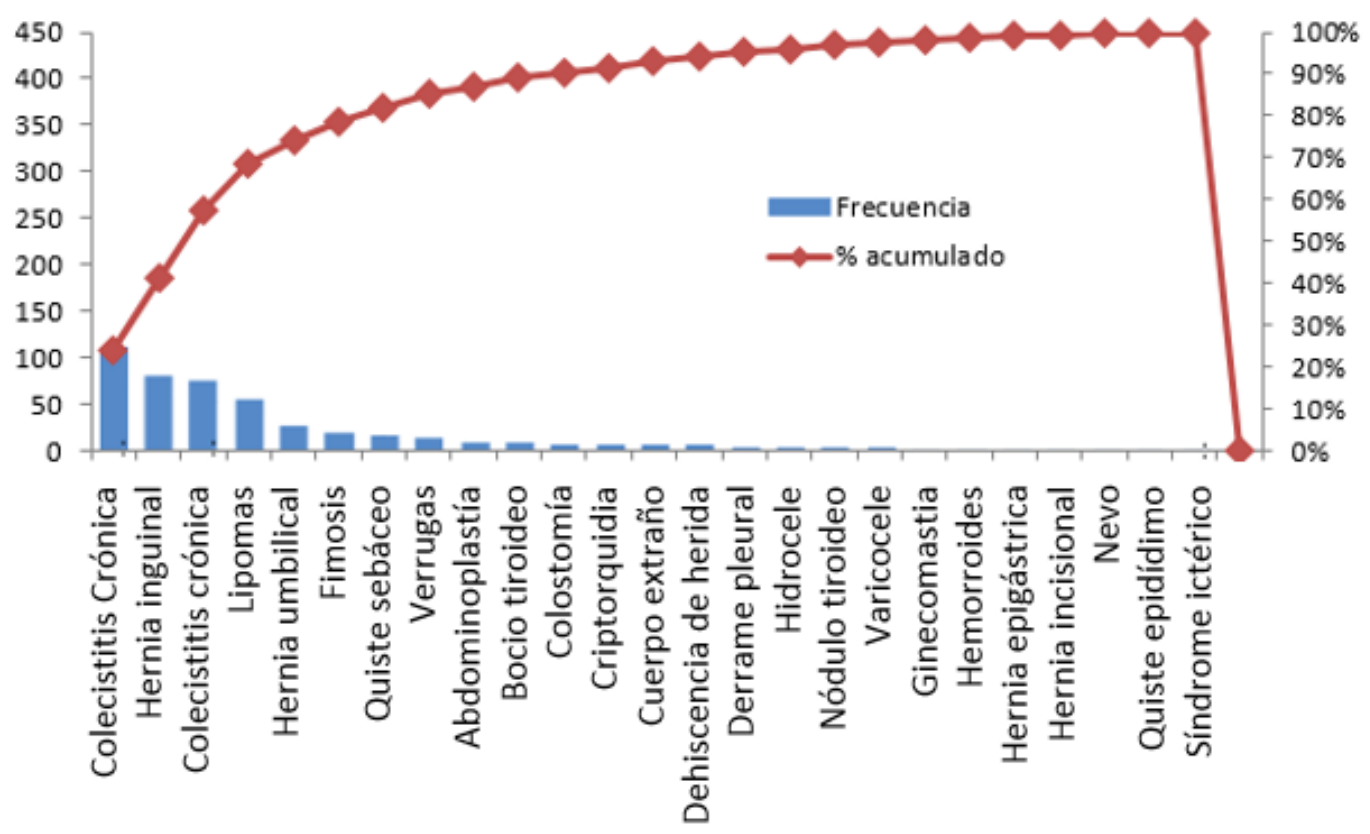

Figura 1. Diagrama de Pareto de las cirugías más comunes

El diagrama mostrado en la Figura 1, arrojó las cirugías que representan el 80\% de todas las intervenciones quirúrgicas selectivas del hospital y a partir de ellas se realizaron los demás análisis estadísticos. Usando los datos históricos de estas cirugías se analizó sí existía una diferencia entre los tiempos de atención por cirujano realizando la prueba ANOVA. Debido a que si existían diferencias, fue necesario realizar pruebas de bondad de ajuste usando el software "Expertfit" por cada cirujano y por cada tipo de cirugía, para 
buscar qué distribución estadística teórica, representaba mejor el comportamiento del tiempo de operación de cada una de ellas. Esta información fue ingresada al simulador. Las técnicas que se usaron para recolectar información y para sustentar los datos cuantitativos fueron la entrevista a personal clave y la revisión y extracción de tiempos de la bitácora de cirugías del hospital.

\section{Resultados}

Aquí se detallan los resultados obtenidos con la información recolectada de las bases de datos del Hospital El Progreso, al igual que la información obtenida por las entrevistas realizadas al personal de cirugía del hospital. Con estos datos se logró hacer un análisis que dio respuesta a los objetivos de investigación.

\subsection{Simulación}

Con la información tabulada en la bitácora de cirugías se pudieron realizar todas las pruebas estadísticas necesarias para su posterior análisis y toma de decisiones en busca de reducir la mora quirúrgica. Entre ellas se logró identificar las distribuciones estadísticas del tiempo de proceso de las cirugías más frecuentes de cada cirujano (80\%). Con estas distribuciones se hicieron las diferentes suposiciones y se corrieron los diferentes escenarios en el software de simulación Flexsim (Flexsim, 2012).

Usando la información obtenida de la bitácora del hospital, se determinó que el Hospital El Progreso solo trabaja en un $88.8 \%$ del tiempo, debido a que el resto es perdido por la suma de diferentes situaciones, como la falta de insumos, huelgas, etc. Esto significa que el hospital trabaja en un año 1108 horas de las 1248 que en teoría debieran trabajarse. Se construyó por lo tanto un modelo en Flexsim que consideraba la entrada de los pacientes tanto selectivos como de emergencia, las colas que naturalmente se hacen frente a la sala de operaciones (mora), la prioridad que tienen las emergencias sobre las cirugías selectivas, los diferentes cirujanos (recursos) con diferentes tiempos de atención y horarios de trabajo o turnos, etc.

Usando los resultados de correr el modelo por un año (tiempo acelerado) 100 veces (réplicas) y usando los datos históricos de la bitácora del número de cirugías de emergencia y selectivas atendidas durante el 2012, se probó estadísticamente la validez del modelo. Los datos obtenidos a través del modelo de la situación actual en cuanto a cantidades de cirugías de emergencia (188 en promedio) y selectivas (489 en promedio) se pueden observar en las Figuras 2 y 3. 
M. J. Sánchez y L.F. Enamorado / Innovare. Vol. 4, Núm. 2 (2015) 41 - 53

\begin{tabular}{|c|c|c|c|c|}
\hline \multicolumn{5}{|c|}{ Summary } \\
& Mean $(90 \%$ Confidence) & Sample Standard Deviation & Min & Max \\
\hline Scenario 1 & $185.45<187.79<190.13$ & 14.03 & 158 & 224 \\
\hline
\end{tabular}

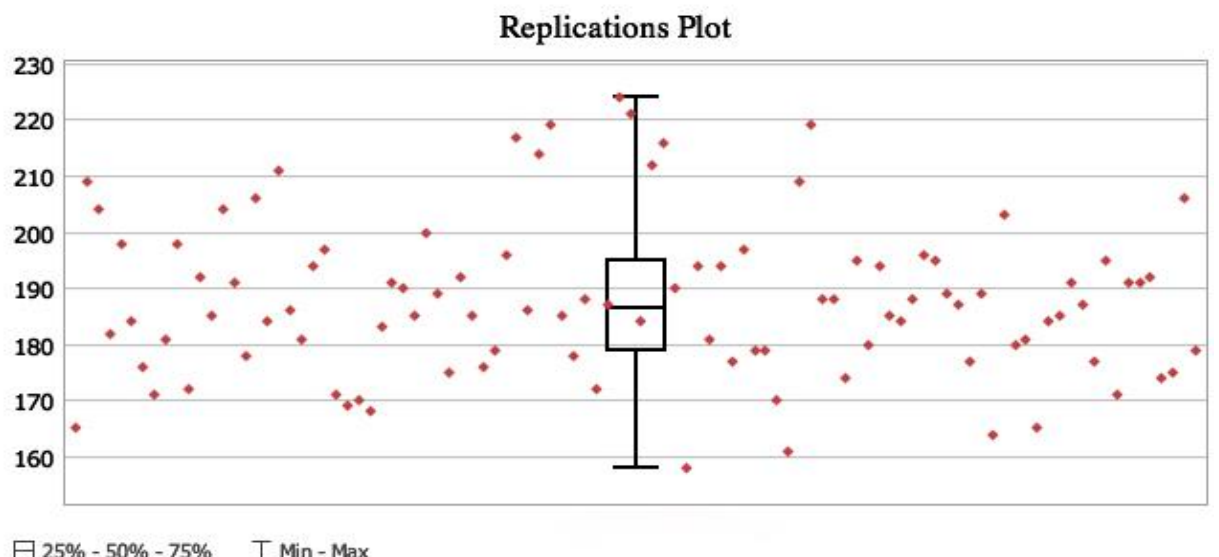

Figura 2 Simulación de cirugías de emergencia de la situación actual del hospital

\begin{tabular}{|c|c|c|c|c|}
\hline \multicolumn{5}{|c|}{ Summary } \\
& Mean $(90 \%$ Confidence) & Sample Standard Deviation & Min & Max \\
\hline Scenario 1 & $484.99<489.26<493.53$ & 25.63 & 396 & 548 \\
\hline
\end{tabular}

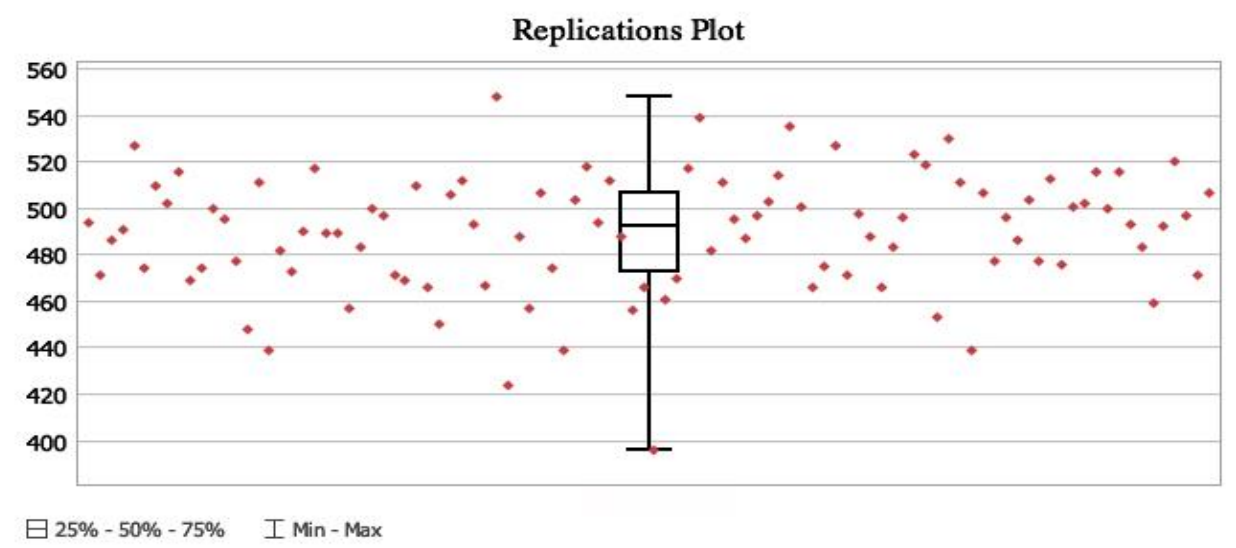

Figura 3 Simulación de cirugía selectiva en la situación actual del hospital

Las Figuras 4 y 5 presentan los resultados de la mora quirúrgica alcanzada por el modelo actual (120 días en promedio) y el total de cirugías realizadas en el año (677 en promedio), todos luego correr el modelo 100 veces con un $90 \%$ de confianza. 
M. J. Sánchez y L.F. Enamorado / Innovare. Vol. 4, Núm. 2 (2015) 41 - 53

\begin{tabular}{|c|c|c|c|c|}
\hline \multicolumn{5}{|c|}{ Summary } \\
Mean (90\% Confidence) & Sample Standard Deviation & Min & Max \\
\hline Scenario 1 & $119.1<120.46<121.82$ & 8.2 & 102.61 & 149.57 \\
\hline
\end{tabular}

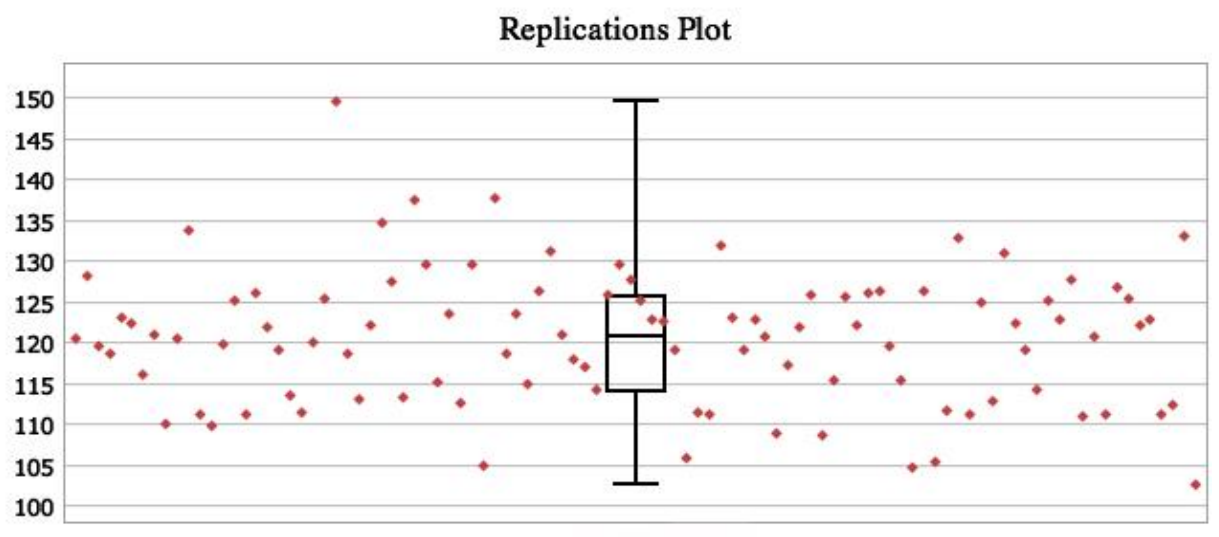

日 $25 \%-50 \%-75 \%$ 工 Min-Max

Figura 4 Simulación de la mora quirúrgica en el Hospital El Progreso

\begin{tabular}{|c|c|c|c|c|}
\hline \multicolumn{5}{|c|}{ Summary } \\
& Mean (90\% Confidence) & Sample Standard Deviation & Min Max \\
\hline Scenario 1 & $673.18<677.05<680.92$ & 23.24 & 580 & 725 \\
\hline
\end{tabular}

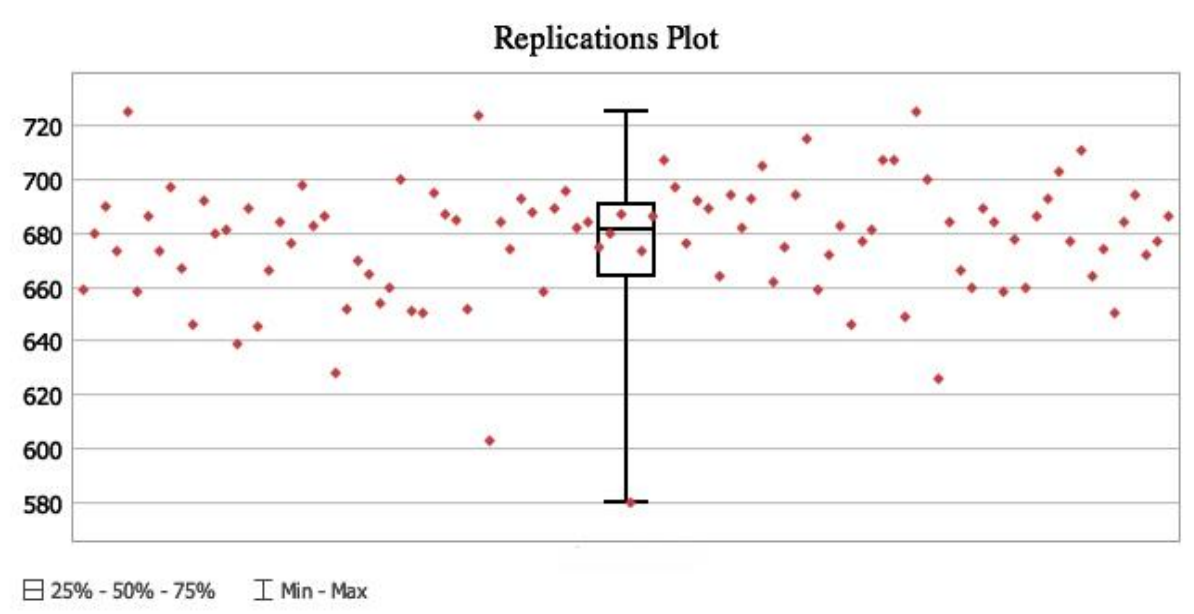

Figura 5 Simulación de la salida de pacientes quirúrgicos

Para poder probar sí este modelo era válido, se realizó la siguiente prueba estadística t: 
Tabla 1 Prueba t de hipótesis para validar modelo de simulación.

\begin{tabular}{|c|c|}
\hline Hipótesis Nula $\mu=$ & 120 \\
\hline Nivel de Significancia & 0.05 \\
\hline Tamaño de la Muestra & 100 \\
\hline Media de la Muestra & 120.46 \\
\hline Desviación Estándar de la muestra & 8.2 \\
\hline Error Estándar de la Media & 0.82 \\
\hline Grados de libertad & 99 \\
\hline Prueba de $t$ Estadística & 0.56097561 \\
\hline Valor Critico Inferior & - \\
\hline Valor Critico Superior & 1.984216952 \\
\hline$p$-Valor & 0.5760816952 \\
\hline No Rechaza la Hipótesis Nula & \\
\hline
\end{tabular}

Mediante esta prueba de hipótesis se concluye que el modelo de simulación es válido ya que se probó que este arroja valores de mora quirúrgica estadísticamente iguales al valor promedio obtenido durante el año 2012. Ya con el modelo validado se plantearon diferentes escenarios para ver el impacto que estos tenían en la mora quirúrgica en el Hospital El Progreso y determinar cuál es el más efectivo en costo y reducción.

\subsection{Escenario 1 - Habilitar una segunda sala de operación.}

En el primer escenario se habilitó una segunda sala de operaciones para mejorar la eficiencia en el turno de la mañana hay dos cirujanos disponibles. Esta segunda sala ya se encuentra acondicionada para realizar todo tipo de cirugías, por lo que solo habrá que reasignar personal de apoyo para que pueda ser utilizada. A continuación se muestra el efecto que este cambio tiene en la mora quirúrgica y en otras variables de interés.

Tabla 2 Resultados del escenario 1

\begin{tabular}{|c|c|c|c|c|c|c|c|c|}
\hline Parámetro & \multicolumn{7}{|c|}{ Resumen } & \\
\hline $\begin{array}{c}\text { Pacientes } \\
\text { Consulta } \\
\text { Externa }\end{array}$ & \multicolumn{5}{|c|}{ Media (95\% Confianza) } & $\begin{array}{c}\text { Desviación } \\
\text { Estándar }\end{array}$ & Min & Max \\
\cline { 2 - 9 } & 2,484 & $<$ & 2,490 & $<$ & 2,496 & 46 & 2,378 & 2,626 \\
\hline Mora (Tiempo) & 15,343 & $<$ & 15,739 & $<$ & 16,135 & 2,983 & 8,850 & 29,643 \\
\hline P. Emergencias & 188 & $<$ & 190 & $<$ & 192 & 15 & 152 & 235 \\
\hline P. Selectivos & 810 & $<$ & 813 & $<$ & 816 & 22 & 744 & 864 \\
\hline Total de cirugías & 1,000 & $<$ & 1,003 & $<$ & 1,007 & 25 & 930 & 1,059 \\
\hline Mora (Días) & 37 & $<$ & 38 & $<$ & 39 & 7 & 22 & 72 \\
& & & & & & & & \\
\hline
\end{tabular}

Con un $95 \%$ de confianza, en el parámetro de pacientes de consulta externa se obtuvo una media de entre 2,484 y 2,496 pacientes, con una desviación estándar de 46. En el parámetro de mora (desde el punto de vista de tiempo de espera) se obtuvo una media de entre 15,343 y 16,135 minutos por paciente, con una desviación estándar de 2983, lo que al convertirlo a días ( 7 horas diarias de trabajo) se convierte en un promedio de entre 37 y 39 días. En el parámetro de pacientes emergencias, se obtuvo una media de entre 188 y 192 pacientes, con una desviación estándar de 15. En el parámetro de pacientes selectivos se obtuvo una media entre 810 y 816 pacientes con una desviación estándar de 22 . En el parámetro de total de cirugías se obtuvo en promedio entre 1,000 y 1,007, con una desviación estándar de 25 cirugías. 


\subsection{Escenario 2 - Reducir $10 \%$ el tiempo perdido por demás variables}

Para el segundo escenario se planteó reducir en un $10 \%$ el tiempo que se pierde por variables como huelgas, falta de insumos, etc. Esta mejora representaría un $11.8 \%$ de más tiempo de operación, que representaría 140 minutos. Al reducir este tiempo muerto se espera mejorar la eficiencia del hospital, por lo que este escenario busca determinar cómo esta mejora afectaría la mora quirúrgica del hospital. El efecto se ve reflejado a continuación en la simulación:

Tabla 1 Resultados del escenario 2

\begin{tabular}{|c|c|c|c|c|c|c|c|c|}
\hline Parámetro & \multicolumn{8}{|c|}{ Resumen } \\
\hline \multirow{2}{*}{$\begin{array}{c}\text { Pacientes Consulta } \\
\text { Externa }\end{array}$} & \multicolumn{5}{|c|}{ Media (95\% Confianza) } & \multirow{2}{*}{$\begin{array}{c}\begin{array}{c}\text { Desviación } \\
\text { Estándar }\end{array} \\
50\end{array}$} & \multirow{2}{*}{$\begin{array}{c}\text { Min } \\
2,607\end{array}$} & \multirow{2}{*}{$\begin{array}{c}\text { Max } \\
2,876 \\
\end{array}$} \\
\hline & 2,730 & $<$ & 2,737 & $<$ & 2,744 & & & \\
\hline Cirugías Emergencias & 207 & $<$ & 209 & $<$ & 211 & 15 & 173 & 253 \\
\hline P. Selectivos & 534 & $<$ & 537 & $<$ & 541 & 27 & 439 & 604 \\
\hline Total Cirugías & 743 & $<$ & 746 & $<$ & 749 & 23 & 648 & 803 \\
\hline Mora (Días) & 125 & $<$ & 126 & $<$ & 127 & 9 & 108 & 155 \\
\hline
\end{tabular}

Con un $95 \%$ de confianza, en el parámetro de pacientes de consulta externa se obtuvo una media de entre 2,730 y 2,744 pacientes, con una desviación estándar de 50. En el parámetro de cirugías de emergencias, se obtuvo una media de entre 207 y 211 cirugías, con una desviación estándar de 15. En el parámetro de pacientes selectivos se obtuvo una media de entre 534 pacientes y 541, con una desviación estándar de 27. En el parámetro de total de cirugías, se obtuvo en promedio entre 743 y 749, con una desviación estándar de 23 cirugías y por último, en la mora en días se obtuvo en promedio de entre 125 y 127 días, con una desviación estándar de 9.

\subsection{Escenario 3 - Reducir en un $10 \%$ el tiempo promedio de operación}

Para el tercer escenario, se consideró simular que sucedería si se lograra una reducción del $10 \%$ en los tiempos promedios de operación de las cirugías realizadas por los tres cirujanos. Se propone obtener esta reducción en los tiempos de cirugía producto del uso de herramientas de mejora para eficientar los procesos que los cirujanos siguen para las diferentes intervenciones que realizan.

Tabla 4 Resultados del escenario 3

\begin{tabular}{|c|c|c|c|}
\hline \multicolumn{4}{|c|}{ Escenario Actual } \\
\hline & $\begin{array}{c}\text { Media (95\% } \\
\text { Confianza) }\end{array}$ & Min & Max \\
\hline $\begin{array}{c}\text { Pacientes } \\
\text { Consulta } \\
\text { Externa }\end{array}$ & 2492.91 & 2327 & 2652 \\
\hline $\begin{array}{c}\text { Pacientes } \\
\text { Emergencia }\end{array}$ & 188.81 & 157 & 226 \\
\hline $\begin{array}{c}\text { Pacientes } \\
\text { Selectivos }\end{array}$ & 494.6 & 435 & 553 \\
\hline Salidas & 683.41 & 626 & 730 \\
\hline $\begin{array}{c}\text { Mora } \\
\text { (Tiempo) }\end{array}$ & 49021.3 & 40593.7 & 61681 \\
\hline mora (Días) & 119 & 99 & 150 \\
\hline
\end{tabular}

\begin{tabular}{|c|c|c|c|}
\hline \multicolumn{4}{|c|}{ Escenario Incremento 10\% Eficiencia } \\
\hline & $\begin{array}{c}\text { Media (95\% } \\
\text { Confianza) }\end{array}$ & Min & Max \\
\hline $\begin{array}{c}\text { Pacientes } \\
\text { Consulta } \\
\text { Externa }\end{array}$ & 2490.86 & 2377 & 2632 \\
\hline $\begin{array}{c}\text { Pacientes } \\
\text { Emergencia }\end{array}$ & 190.5 & 152 & 226 \\
\hline $\begin{array}{c}\text { Pacientes } \\
\text { Selectivos }\end{array}$ & 528.16 & 389 & 609 \\
\hline Salidas & 718.66 & 603 & 790 \\
\hline $\begin{array}{c}\text { Mora } \\
\text { (Tiempo) }\end{array}$ & 45987.9 & 37250.2 & 56195.7 \\
\hline mora (Días) & 112 & 91 & 137 \\
\hline
\end{tabular}


Como se observa en la tabla 4, a pesar de que con la reducción del tiempo de operación hay un incremento en la eficiencia en las operaciones quirúrgicas, el impacto que esto tiene en la mora quirúrgica en el Hospital El Progreso no es tan significativo como se esperaba.

Con un $95 \%$ de confianza, se redujo la mora quirúrgica en promedio de 119 a 112 días (7 días menos), lo cual representa casi un 6\% de mejora en eficiencia. Del análisis realizado, producto de los registros de los tiempos de inicio y finalización de las cirugías durante el corto período de un año (2012), se encontraron posibles factores influyentes. De ese análisis se encontró que la técnica de anestesia y las especialidades de cada cirugía son factores de mucha influencia. Sin embargo, la pobre planificación y el deficiente proceso de programación de las citas, así como la falta de compromiso del personal de todo nivel, resultan ser los factores más importantes en la mora de cirugía que actualmente tiene el Hospital El Progreso.

\subsection{Escenario 4 - Combinacion de los primeros tres escenarios}

Para este escenario se buscó combinar los primeros tres escenarios y ver el impacto que esto tiene en la mora quirúrgica del hospital. El habilitar una segunda sala de operación, mejorar en un $10 \%$ la eficiencia en los tiempos promedios de operaciones y reducir en un $10 \%$ los tiempos perdidos, da como resultado en la simulación los siguientes datos:

Tabla 5 Resultados del escenario 4

\begin{tabular}{|c|c|c|c|c|c|c|c|c|}
\hline Parámetro & \multicolumn{7}{c|}{ Resumen } \\
\hline $\begin{array}{c}\text { Pacientes } \\
\text { Consulta } \\
\text { Externa }\end{array}$ & \multicolumn{3}{|c|}{ Media (95\% Confianza) } & $\begin{array}{c}\text { Desviación } \\
\text { Estándar }\end{array}$ & Min & Max \\
\cline { 2 - 9 } & 2728 & $<$ & 2734 & $<$ & 2741 & 51 & 2599 & 2887 \\
\hline Mora (Tiempo) & 10951 & $<$ & 11203 & $<$ & 11413 & 1894 & 7229 & 18421 \\
\hline P. Emergencias & 207 & $<$ & 209 & $<$ & 211 & 14 & 154 & 244 \\
\hline P. Selectivos & 875 & $<$ & 878 & $<$ & 881 & 26 & 813 & 945 \\
\hline Salida & 1083 & $<$ & 1087 & $<$ & 1091 & 30 & 1005 & 1158 \\
\hline Mora (Días) & 27 & $<$ & 27 & $<$ & 28 & 5 & 18 & 45 \\
\hline
\end{tabular}

Con un $95 \%$ de confianza, la cantidad de pacientes de consulta externa atendidos resultó ser de entre 2,728 y 2,741 pacientes con una desviación estándar de 51 pacientes. En este caso, la mora de tiempo es de entre 27 y 28 días con una desviación estándar de 5 días. Los pacientes de emergencia atendidos fueron entre 207 y 211, con una desviación estándar de 14.

\subsection{Escenario 5 - Habilitar segunda sala para cirugias de emergencia}

En el último escenario se buscó ver el efecto que tiene en la mora quirúrgica la habilitación de una segunda sala de operaciones dedicada exclusivamente para cirugías de emergencias. Esto se hizo debido a que las emergencias son un gran factor que interrumpe las cirugías selectivas programadas. Por lo que, en la tabla 6 se muestra el resultado de este escenario. 
M. J. Sánchez y L.F. Enamorado / Innovare. Vol. 4, Núm. 2 (2015) 41 - 53

Tabla 6 Resultados del escenario 5

\begin{tabular}{|c|c|c|c|c|c|c|c|c|}
\hline \multirow{3}{*}{\begin{tabular}{|c|} 
Parámetro \\
$\begin{array}{c}\text { Pacientes Consulta } \\
\text { Externa }\end{array}$
\end{tabular}} & \multicolumn{8}{|c|}{ Resumen } \\
\hline & \multicolumn{5}{|c|}{ Media (95\% Confianza) } & \multirow{2}{*}{$\begin{array}{c}\text { Desviación } \\
\text { Estándar } \\
46\end{array}$} & \multirow{2}{*}{$\frac{\text { Min }}{2,378}$} & \multirow{2}{*}{$\frac{\text { Max }}{2,648}$} \\
\hline & 2.490 & $<$ & 2.499 & $<$ & 2.508 & & & \\
\hline $\begin{array}{c}\text { Cirugías } \\
\text { Emergencias }\end{array}$ & 189 & $<$ & 192 & $<$ & 195 & 14 & 143 & 230 \\
\hline Pacientes Selectivos & 680 & $<$ & 684 & $<$ & 687 & 18 & 644 & 721 \\
\hline Total Cirugías & 872 & $<$ & 876 & $<$ & 881 & 23 & 821 & 931 \\
\hline Mora (Días) & 76 & $<$ & 77 & $<$ & 79 & 7 & 61 & 98 \\
\hline
\end{tabular}

Con un 95\% de confianza, el parámetro de pacientes de consulta externa atendidos estuvo en un intervalo de 2,490 a 2,508 pacientes con una desviación estándar de 46. En el parámetro de cirugías de emergencias se obtuvo una media de entre 189 y 195 cirugías con una desviación estándar de 14. En el parámetro de pacientes selectivos se obtuvo una media de 680 a 687 pacientes con una desviación estándar de 18. En el parámetro de total de cirugías se obtuvo en promedio entre 872 y 881 con una desviación estándar de 23 cirugías y por último en la mora en días se obtuvo en promedio de 76 a 79 días con una desviación estándar de 7.

\subsection{Estudio financiero}

Como a partir de las simulaciones realizadas se conoce el impacto que cada escenario tiene con relación a la mora quirúrgica, se hizo un análisis de costo beneficio para tomar la decisión sobre cuál escenario implementar, tomando en cuenta los recursos que posee el Hospital El Progreso. El escenario uno, incurre en un costo de L 327,600.00 anuales. Esto es por el pago de planilla de dos instrumentistas y un anestesista, ya que el salario mensual de las instrumentistas es de L 7,200.00 mensuales y el del anestesista es de L $9,000.00$. Cabe mencionar que este personal será utilizado los días martes y jueves, en jornada matutina, para así tener dos quirófanos en funcionamiento, siempre y cuando no haya ausencias del personal permanente del hospital.

El escenario dos no presenta costos para el hospital, ya que para poder reducir en $10 \%$ los días inactivos en el hospital (falta de insumos, cancelación de pabellón quirúrgico, asambleas informativas, falta de personal técnico, etc.) se aplicó el reglamento interno del hospital que está basado en el código del trabajo y los diferentes estatutos gremiales. El escenario tres, incurre en un costo anual de L 177,000.00, ya que anualmente se enviará un cirujano a uno de los congresos de cirugía. Dado que el Congreso Centroamericano de Cirugía tiene un costo de L 40,000.00 y el Congreso Nacional de Cirugía tiene un costo de L 10,000.00 y se llevan a cabo cada dos años, sin coincidir en el año de la realización de cada uno, el costo es de L 25,000.00.

Por lo tanto se realizó un promedio de ambos congresos, para poder cuantificarlo en esta investigación. La capacitación será reforzada con un curso práctico (fellowship) que tiene un costo de \$6,000.00 (L 120,000.00) más transporte \$1,600.00 (L 32,000.00) (CENCIL, 2013).

El escenario cuatro, incurre en un costo anual de L 504,600.00, ya que es la suma de los tres primeros escenarios. El escenario cinco, incurre en un costo anual de L 327,600.00 
ya que el segundo quirófano sería usado solo para cirugías de emergencias y se necesitaría el mismo personal que el escenario uno.

En la figura 6, se observa el análisis de costo-beneficio que se realizó a los cinco escenarios planteados en esta investigación, al igual que el costo que tiene la implementación de cada uno. Por lo que el mejor escenario es el primero, ya que es el que más se acerca al ideal de realizarlo con un bajo costo y un alto impacto (disminución en la mora quirúrgica). Asimismo, se estaría cumpliendo con lo indicado por la OMS en días de mora después que el paciente es visto en consulta externa y cumpliendo con el presupuesto asignado al hospital para operar.

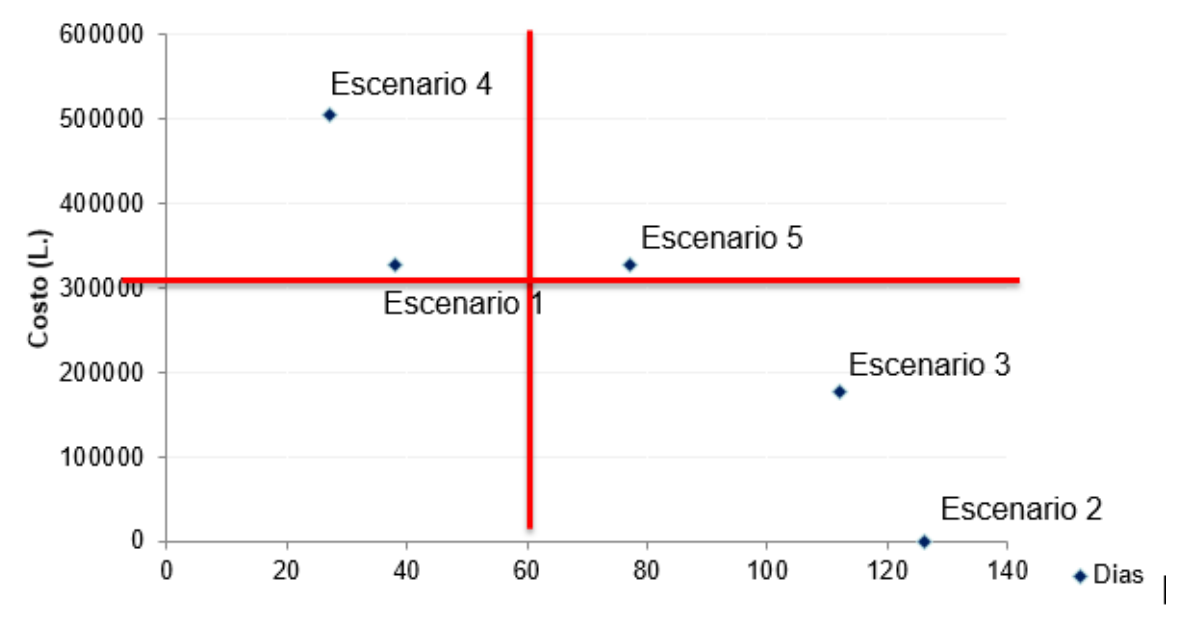

Figura 6 Análisis costo - beneficio de la implementación de los escenarios

\section{Conclusiones}

Las conclusiones de la presente investigación son las siguientes:

1) Se acepta la hipótesis nula, lo que hace válida la simulación de la situación actual del hospital. Esto, a su vez, hace válidos los escenarios planteados y el impacto con relación a la mora quirúrgica.

2) El modelo de simulación del escenario 4, indica que es posible reducir la mora quirúrgica del valor actual de 120 días al valor aceptado por el indicador de la OMS, que es de 30 días, ya que el valor que proyecta la simulación indica un valor de 27 días. El escenario 4 es la combinación de habilitar una segunda sala de cirugía, más una reducción del 10\% del tiempo quirúrgico por los cirujanos y la reducción del $10 \%$ de las otras variables.

3) El mejor escenario para implementar en el Hospital El Progreso, es el escenario 1 (implementar una segunda sala de operación), ya que su impacto es alto (38 días de mora) y su costo es bajo (Lps. 327,600.00).

4) Se puede concluir a través de pruebas estadísticas (prueba de hipótesis y de caja y bigote) que el cirujano "A", toma más tiempo quirúrgico que los cirujanos "B" y "C".

5) Mediante el estudio de colas, se encontró que la causa principal de cancelación de cirugías selectivas y aumento de mora quirúrgica son las cirugías de emergencia.

6) En la prueba ANOVA de los tres cirujanos se encontró gran variabilidad en los tiempos que les tomaba la misma cirugía según patología quirúrgica, reforzando 
el hecho de que cada paciente es diferente y único, aun si se tienen las mismas patologías de base y secundarias.

7) Al igual que existe una gran variabilidad entre los tiempos de cirugía por los cirujanos, el tiempo de preparación de la sala de operaciones también presenta gran variabilidad por parte de las enfermeras y personal de aseo.

8) El que no haya uso de la pizarra quirúrgica en el hospital, limitó la información obtenida para la construcción de la bitácora, por lo que se pierde información como la cuantificación de otras variables (insumos, asambleas informativas, etc.).

9) Se concluye que el $80 \%$ de las cirugías que se realizan en el Hospital El Progreso se concentran en: Colecistitis crónica calculosa técnica cerrada, hernia inguinal, colecistitis crónica calculosa técnica abierta, fimosis, hernia umbilical y lipomas.

\title{
Bibliografía
}

CENCIL. (2013). Centro de entrenamiento de cirugía laparoscópica. http://www.cencilcorrientes.com.ar /curso-universitario-hands-on/fellowship.php

Flexsim (2012). ¿Qué es Flexsim? Obtenido en: http://www.flexsim.cl/about.php

Hernández, R., Fernández, C., \& Baptista, P. (2006). Metodología de la investigación. México, DF.: McGraw-Hill Interamericana.

Rojas, L., Vergara Matamala, E., (13 de Julio de 2012). Una formulación matemática y de solución para programar cirugías con restricciones de recursos humanos en el hospital público. Ingeniare. Revista chilena de ingeniería, vol. 20 No 2, 2012, pp. 230-241.

Schwartz. (2010). Principios de Cirugía, (9º edición): Mc graw-Hill.

\author{
"LA REVISTA INNOVARE NO SE HACE RESPONSABLE EN \\ NINGÚN CASO DE LOS CONTENIDOS, DATOS, CONCLUSIONES \\ U OPINIONES VERTIDAS EN LOS ARTÍCULOS PUBLICADOS, \\ SIENDO ESTA RESPOSABILIDAD EXCLUSIVA DEL (DE LOS) \\ AUTOR (AUTORES)"
}

